\title{
Fractional Hankel and Bessel wavelet transforms of almost periodic signals
}

\section{Banu Ünalmış Uzun*}

\section{${ }^{*}$ Correspondence:} buzun@isikun.edu.tr

Department of Mathematics, Işık University, Şile, Istanbul, 34980, Turkey

\begin{abstract}
The main objective of this paper is to study the Hankel, fractional Hankel, and Bessel wavelet transforms using the Parseval relation. We construct a generalized frame and write new relations and inequalities using almost periodic functions, strong limit power signals, and these transform methods.
\end{abstract}

MSC: 42A75; 42A24; 42A38

Keywords: almost periodic function; fractional Hankel transform; Bessel wavelet transform; frame

\section{Introduction}

The Hankel transform (HT) is a well-known integral transform which uses the $v$ th order Bessel function of the first kind, $J_{v}$, as a kernel. Since the HT is equivalent to the twodimensional Fourier transform (FT) of a circularly symmetric function, it plays an important role in a number of applications including optical data processing, digital filtering, etc. [1-3].

The conventional HT is extended to the fractional Hankel transform (FrHT) by Kerr [4] and Namias [5]. Its properties are discussed in detail by several authors [6-9] and its applications in many areas (such as optics, signal processing, quantum mechanics) are given in [10-12]. Using the theory of Hankel translation, Pathak and Dixit defined continuous and discrete Bessel wavelet transforms (BWT) and studied their properties [13]. The fractional Hankel transformation and the continuous fractional Bessel wavelet transformation, some of their basic properties and applications are studied in [14]. In [15], the relation between the Bessel wavelet transformation and the Hankel-Hausdorff operator is discussed.

In this paper, we first of all introduce the FrHT, the BWT, and almost periodic functions and some of their properties in brief. A generalized frame decomposition for almost periodic functions is constructed by using an orthogonal basis with Laguerre functions relating to the FrHT. We also give various relations using the HT, FrHT, BWT, and strong limit power signals.

\subsection{Hankel and fractional Hankel transforms}

We define the Fourier transform of an integrable function $f$ as

$$
\hat{f}(t)=(\mathcal{F} f)(t)=\frac{1}{\sqrt{2 \pi}} \int_{-\infty}^{\infty} f(x) e^{-i t x} d x .
$$

(c) 2015 Ünalmış Uzun. This article is distributed under the terms of the Creative Commons Attribution 4.0 International License (http://creativecommons.org/licenses/by/4.0/), which permits unrestricted use, distribution, and reproduction in any medium, provided you give appropriate credit to the original author(s) and the source, provide a link to the Creative Commons license, and indicate if changes were made. 
The general HT is given as

$$
\mathcal{H}_{v}[f](x)=\int_{0}^{\infty} y J_{v}(y x) f(y) d y, \quad \operatorname{Re}(v)>-\frac{1}{2}
$$

and the inverse is given by

$$
f(y)=\int_{0}^{\infty} x \mathcal{H}_{v}[f](x) J_{v}(y x) d x
$$

where $J_{v}$ is the $v$ th order Bessel function of the first kind [16].

The HT of order zero is an integral transform equivalent to a two-dimensional FT with a symmetric integral kernel,

$$
\begin{aligned}
\mathcal{H}_{0}[f](q) & =\int_{0}^{\infty} r J_{0}(q r) f(r) d r \\
& =\int_{0}^{\infty}\left(\frac{1}{2 \pi} \int_{0}^{2 \pi} e^{-i r q \cos \theta} d \theta\right) r f(r) d r \\
& =\frac{1}{2 \pi} \int_{0}^{\infty} \int_{-\phi}^{2 \pi-\phi} e^{-i r q \cos \theta} r f(r) d r d \theta \\
& =\frac{1}{2 \pi} \int_{0}^{\infty} \int_{0}^{2 \pi} e^{-i r q \cos (\theta-\phi)} r f(r) d r d \theta \\
& =\frac{1}{2 \pi} \int_{-\infty}^{\infty} \int_{-\infty}^{\infty} f(x, y) e^{-i(u x+v y)} d x d y \\
& =\hat{f}(u, v),
\end{aligned}
$$

where $r=\sqrt{x^{2}+y^{2}}$ and $q=\sqrt{u^{2}+v^{2}}$. This is also known as the Fourier-Bessel transform. Also Parseval's theorem holds for the HT:

$$
\int_{0}^{\infty} x \mathcal{H}_{\nu}[f](x) \mathcal{H}_{v}[g](x) d x=\int_{0}^{\infty} y f(y) g(y) d y .
$$

Namias [5] introduced the concept of Fourier transform and Hankel transform of fractional order (FrFT and FrHT), opening the new period of fractional transforms. FrFT with angle $\alpha$ of a signal $f(x)$ is given as

$$
F_{\alpha}(y)=\frac{e^{i\left(\frac{\pi}{4}-\frac{\alpha}{2}\right)}}{\sqrt{2 \pi \sin \alpha}} \int_{0}^{\infty} e^{-i \frac{x^{2}+y^{2}}{2} \cot \alpha+i x y \csc \alpha} f(x) d x
$$

and the inverse is

$$
f(x)=\frac{e^{-i\left(\frac{\pi}{4}-\frac{\alpha}{2}\right)}}{\sqrt{2 \pi \sin \alpha}} \int_{0}^{\infty} e^{i \frac{x^{2}+y^{2}}{2} \cot \alpha-i x y \csc \alpha} F_{\alpha}(y) d y .
$$

The Parseval relation for FrFT is given as

$$
\int_{0}^{\infty}|f(x)|^{2} d x=\int_{0}^{\infty}\left|F_{\alpha}(y)\right|^{2} d y
$$


The FrHT is defined by

$$
\mathcal{H}_{v}^{\alpha}[f](y)=\int_{0}^{\infty} f(x) K_{\alpha}(x, y) d x
$$

where

$$
K_{\alpha}(x, y)=A_{\nu, \alpha} x e^{-\frac{i}{2}\left(x^{2}+y^{2}\right) \cot \frac{\alpha}{2}} J_{\nu}\left(\frac{x y}{\sin \frac{\alpha}{2}}\right)
$$

with

$$
A_{v, \alpha}=\frac{e^{i(v+1)\left(\frac{\pi}{2}-\frac{\alpha}{2}\right)}}{\sin \frac{\alpha}{2}} .
$$

The inverse is given by

$$
f(x)=\int_{0}^{\infty} \mathcal{H}_{v}^{\alpha}[f](y) \overline{K_{\alpha}(x, y)} d y
$$

where

$$
\overline{K_{\alpha}(x, y)}=\frac{e^{-i(v+1)\left(\frac{\pi}{2}-\frac{\alpha}{2}\right)}}{\sin \frac{\alpha}{2}} x e^{\frac{i}{2}\left(x^{2}+y^{2}\right) \cot \frac{\alpha}{2}} J_{v}\left(\frac{x y}{\sin \frac{\alpha}{2}}\right)
$$

as in [17].

\subsection{Orthogonal basis with Laguerre functions}

Let $L_{n}^{v}$ be the generalized Laguerre polynomials defined by means of the Rodrigues formula

$$
L_{n}^{v}(x)=\frac{1}{n !} e^{x} x^{-v} D^{n}\left[e^{-x} x^{n+\nu}\right], \quad n \in \mathbb{N}_{0} \equiv \mathbb{N} \cup\{0\}, x>0
$$

which gives

$$
L_{n}^{v}(x)=\sum_{k=0}^{n}(-1)^{k} \frac{(n+v) !}{(n-k) !(v+k) ! k !} x^{k}
$$

in power series form. The Laguerre functions are defined as

$$
l_{n}^{\nu}(x)=\mathbf{1}_{[0, \infty]}(x) e^{-x / 2} x^{\nu / 2} L_{n}^{v}(x)
$$

and they form an orthogonal basis for the space $L_{2}(0, \infty)$. Let us set $\mathcal{F} S_{n}^{\nu}(x)=l_{n}^{\nu}(x)$ and take $(a)_{n}=a(a+1)(a+2) \ldots(a+n-1)$. It was shown that

$$
S_{n}^{v}(x)=\frac{\Gamma\left(\frac{v}{2}+1\right)(1+v)_{n}}{n !} \sum_{k=0}^{n} \frac{(-n)_{k}\left(\frac{v}{2}+1\right)_{k}}{k !(v+1)_{k}}\left(\frac{1}{\frac{1}{2}-i x}\right)^{k+\frac{v}{2}+1}
$$

and the $S_{n}^{v}$ are orthogonal on the real line [18]. 
The functions $S_{n}^{\nu}$ can be written as a linear combination of Paul's wavelets,

$$
\psi_{\alpha}(x)=\left(\frac{1}{x+i}\right)^{\alpha+1}
$$

such that

$$
S_{n}^{v}\left(\frac{x}{2}\right)=C \sum_{k=0}^{n} c_{k} \psi_{k+\frac{v}{2}}(x)
$$

where the constants $C$ and the coefficients $c_{k}$ defined as

$$
C=\frac{\Gamma\left(\frac{v}{2}+1\right)(1+v)_{n}}{n !}
$$

and

$$
c_{k}=(2 i)^{k+\frac{v}{2}+1} \frac{(-n)_{k}\left(\frac{v}{2}+1\right)_{k}}{k !(v+1)_{k}} .
$$

$S_{n ; a, b}^{\nu}(x)=2^{a / 2} S_{n}^{\nu}\left(2^{a} x-b\right)$ is the natural discretization of $S_{n}^{\nu}(x)$ for every $a, b \in \mathbb{Z}$.

If another complete orthogonal basis $\left\{\psi_{n}^{\nu}\right\}_{n=0}^{\infty}$, given by

$$
\psi_{n}^{v}(x)=x^{v} e^{-\frac{x^{2}}{2}} L_{n}^{v}\left(x^{2}\right)
$$

is chosen, we get

$$
H_{v}\left[y^{v} e^{-\frac{y^{2}}{2}} L_{n}^{v}\left(y^{2}\right)\right](x)=e^{i n \pi} x^{v} e^{-\frac{x^{2}}{2}} L_{n}^{v}\left(x^{2}\right), \quad n \in \mathbb{N}_{0}, x>0,
$$

using equation 7.421(4) in [19]. Then the integral representation

$$
\mathcal{H}_{\nu}^{\alpha}[f](x)=A_{v, \alpha} \int_{0}^{\infty} y e^{-\frac{i}{2}\left(x^{2}+y^{2}\right) \cot \frac{\alpha}{2}} J_{\nu}\left(\frac{x y}{\sin \frac{\alpha}{2}}\right) f(y) d y,
$$

with

$$
A_{v, \alpha}=\frac{e^{i(v+1)\left(\frac{\pi}{2}-\frac{\alpha}{2}\right)}}{\sin \frac{\alpha}{2}},
$$

which is known as the FrHT is obtained [5].

\subsection{Continuous Bessel wavelet transform}

Let

$$
\sigma(x)=\frac{x^{2 \gamma+1}}{2^{\gamma+1 / 2} \Gamma\left(\gamma+\frac{3}{2}\right)}
$$

and

$$
j(x)=C_{\gamma} x^{1 / 2-\gamma} J_{\gamma-1 / 2}(x), \quad C_{\gamma}=2^{\gamma-1 / 2} \Gamma\left(\gamma+\frac{1}{2}\right),
$$


where $\gamma$ is a positive real number and $J_{\gamma-1 / 2}(x)$ is the Bessel function of the first kind of order $\gamma-\frac{1}{2}$. Define

$$
\begin{aligned}
D(x, y, z) & =\int_{0}^{\infty} j(x t) j(y t) j(z t) d \sigma(t) \\
& =2^{3 \gamma-5 / 2}\left[\Gamma\left(\gamma+\frac{1}{2}\right)\right]^{2}\left[\Gamma(\gamma) \pi^{1 / 2}\right]^{-1}(x y z)^{-2 \gamma+1}[\triangle(x, y, z)]^{2 \gamma-2},
\end{aligned}
$$

where $\triangle(x, y, z)$ denotes the area of a triangle with sides $x, y, z$ if such a triangle exists and is zero otherwise. $\triangle(x, y, z)$ is nonnegative and symmetric in $x, y, z$.

$L_{\sigma}^{p}(0, \infty), 1 \leq p<\infty$ is the space of measurable functions $\phi$ on $(0, \infty)$, such that

$$
\begin{aligned}
& \|\phi\|_{p, \sigma}=\left[\int_{0}^{\infty}|\phi(x)|^{p} d \sigma(x)\right]^{1 / p}<\infty, \quad 1 \leq p<\infty, \\
& \|\phi\|_{\infty, \sigma}=\underset{0<x<\infty}{\operatorname{esssup}}|\phi(x)|<\infty .
\end{aligned}
$$

Let $\psi \in L_{\sigma}^{p}(0, \infty), 1 \leq p<\infty$ be given. The Bessel wavelet is given by

$$
\psi_{b, a}(x):=a^{-2 \gamma-1} \int_{0}^{\infty} D(b / a, x / a, z) \psi(z) d \sigma(z)
$$

where $a>0$ and $b \geq 0$. If $\psi \in L_{\sigma}^{2}(0, \infty)$, it satisfies the admissibility condition (see [15])

$$
C_{\mu, \psi}=\int_{0}^{\infty} x^{-2 \mu-2}\left|\mathcal{H}_{\nu}[\psi](x)\right|^{2} d x<\infty, \quad \mu \geq-\frac{1}{2}
$$

where $\mathcal{H}_{v}[\psi](x)$ is the Hankel transform of $\psi(t)$.

Using the Bessel wavelet, Pathak and Dixit [13] introduced the continuous Bessel wavelet transform (BWT) as

$$
\begin{aligned}
\left(B_{\psi} f\right)(b, a) & =\left\langle f, \psi_{b, a}(t)\right\rangle=\int_{0}^{\infty} f(t) \overline{\psi_{b, a}(t)} d \sigma(t) \\
& =a^{-2 \gamma-1} \int_{0}^{\infty} \int_{0}^{\infty} f(t) \overline{\psi(z)} D\left(\frac{b}{a}, \frac{t}{a}, z\right) d \sigma(z) d \sigma(t) .
\end{aligned}
$$

Also Pathak et al. [20] stated the equality

$$
\left(B_{\psi} f\right)(b, a)=(f \sharp \phi)\left(\frac{b}{a}\right), \quad a>0,
$$

using the convolution operator.

\subsection{Almost periodic functions}

The space $\mathcal{A P}$ of almost periodic functions is the closure of quasi-periodic functions in the space $L_{\text {loc }}^{p}$ of $f$, where $\|f\|^{p}$ is locally Lebesgue integrable on $\mathbb{R}$ for $p \geq 1$. This space is defined as the closed subspace of $L^{\infty}(\mathbb{R})$ given as the closed linear span of all functions $e^{i \lambda t}$ where $\lambda \in \mathbb{R}$ (see $[21,22]$ ). Equivalently, it is the completion of the space of trigonometric 
polynomials on $\mathbb{R}$ whose elements can be written as $\sum_{k=1}^{n} a_{k} e^{i \lambda_{k} t}$, where $n \in \mathbb{N}, a_{k} \in \mathbb{C}$, and $\lambda_{k} \in \mathbb{R}$. All $\mathcal{A P}$ functions are uniformly continuous and bounded, and we have

$$
\|f\|_{\mathcal{A P}}^{2}=\lim _{T \rightarrow \infty} \frac{1}{2 T} \int_{-T}^{T}|f(t)|^{2} d t
$$

Let $Q(\mathbb{R})$ consist of functions $q$ in the form

$$
q(t)= \begin{cases}\sum_{l=1}^{m} \lambda_{l} t^{\alpha_{l}}, & t \geq 0, \\ -\sum_{l=1}^{m} \lambda_{l}(-t)^{\alpha_{l},} & t<0,\end{cases}
$$

where $m=1,2, \ldots, \lambda_{l} \in \mathbb{R}, l=1,2, \ldots, m$, and $\alpha_{1}>\alpha_{2}>\cdots>\alpha_{m}>0$. A function of the form

$$
P(t)=\sum_{k=1}^{n} a_{k} e^{i q_{k}(t)}
$$

is called a generalized trigonometric polynomial on $\mathbb{R}$, where $a_{k} \in \mathbb{C}, q_{k}(t) \in Q(\mathbb{R})$, and $k=1,2, \ldots, n$. Denote by $\operatorname{Gtrig}(\mathbb{R})$ the set of all such polynomials.

A function $f$ on $\mathbb{R}$ is said to have a strong limit power if for every $\varepsilon>0$ there exists a $P_{\varepsilon} \in \operatorname{Gtrig}(\mathbb{R})$, such that

$$
\left\|f-P_{\varepsilon}\right\|=\sup \left\{\left|f(t)-P_{\varepsilon}(t)\right|: t \in \mathbb{R}\right\}<\varepsilon .
$$

Denote by $\mathcal{S} \mathcal{L P}(\mathbb{R})$ the set of all such functions. It is obvious that $\mathcal{A P}(\mathbb{R}) \subset \mathcal{S} \mathcal{L P}(\mathbb{R})$. The inner product of the $\mathcal{S} \mathcal{L P}(\mathbb{R})$ space is defined by

$$
\langle f, g\rangle:=\lim _{T \rightarrow \infty} \frac{1}{2 T} \int_{-T}^{T} f(t) \bar{g}(t) d t
$$

(see $[23,24])$.

\section{Main results}

Proposition 1 Let $f \in \mathcal{A P}$. Then

$$
\lim _{T \rightarrow \infty} \frac{1}{2 T} \int_{-T}^{T} \int_{0}^{2 \pi} \int_{0}^{\infty} q\left|\mathcal{H}_{0}[f](q)\right|^{2} d q d \phi d t=\|f\|_{L^{2}(x, y), A P(t)} .
$$

Proof Using the equality (1.2) and the Parseval theorem for the two-dimensional Fourier transform, we get

$$
\int_{0}^{2 \pi} \int_{0}^{\infty} q\left|\mathcal{H}_{0}[f](q)\right|^{2} d q d \phi=\int_{-\infty}^{\infty} \int_{-\infty}^{\infty}|\hat{f}(u, v)|^{2} d u d v=\int_{-\infty}^{\infty} \int_{-\infty}^{\infty}|f(x, y)|^{2} d x d y
$$

and the result follows.

Lemma 1 Let $F_{\frac{\alpha}{2}}(y)$ be in $L^{1}(0, \infty)$ for $v>-1$. Then

$$
H_{v}^{\alpha}\left[e^{-x} f(x)\right]=C_{\alpha} \int_{0}^{\infty} g(y) F_{\frac{\alpha}{2}}(y) d y
$$


where

$$
C_{\alpha}=\frac{e^{i\left[\nu\left(\frac{\pi}{2}-\frac{\alpha}{2}\right)+\frac{\pi}{4}-\frac{\alpha}{4}\right]}}{\left|\sin \frac{\alpha}{2}\right|^{\nu+\frac{3}{2}} \sqrt{2 \pi}}
$$

and

$$
\begin{gathered}
g(y)=\left(1+2 i y \csc \frac{\alpha}{2}\right)^{-\frac{3}{2}}\left(\left(1+i y \csc \frac{\alpha}{2}\right)+v\left(1+2 i y \csc \frac{\alpha}{2}\right)^{\frac{1}{2}}\right) \\
\cdot\left(\frac{y}{\left|\sin \frac{\alpha}{2}\right|\left(1+i y \csc \frac{\alpha}{2}\right)\left(1+2 i y \csc \frac{\alpha}{2}\right)^{\frac{1}{2}}}\right)^{v} .
\end{gathered}
$$

Moreover, if $g(y) \in L^{\infty}$, then the FrHT satisfies

$$
\left|H_{v}^{\alpha}\left[e^{-x} f(x)\right]\right|^{2} \leq\left|C_{\alpha}\right|^{2}\left\|g^{2}\right\|_{\infty}\|f\|_{2} .
$$

Proof Using the definition of the FrHT (1.6) and the inverse FrFT (1.4), we get

$$
\begin{aligned}
\mathcal{H}_{\nu}^{\alpha}\left[e^{-x} f(x)\right]= & A_{v, \alpha} \int_{0}^{\infty} x e^{-x} e^{-i \frac{x^{2}+y^{2}}{2}} \cot \frac{\alpha}{2} J_{\nu}\left(\frac{x y}{\sin \frac{\alpha}{2}}\right) \\
& \cdot \int_{0}^{\infty} \frac{e^{-i\left(\frac{\pi}{4}-\frac{\alpha}{4}\right)}}{\sqrt{2 \pi \sin \frac{\alpha}{2}}} e^{i \frac{x^{2}+y^{2}}{2} \cot \frac{\alpha}{2}-i x y \csc \frac{\alpha}{2}} F_{\frac{\alpha}{2}}(y) d y d x .
\end{aligned}
$$

Changing the order of integration and using Theorem 2.1 in [25],

$$
\begin{aligned}
\mathcal{H}_{v}^{\alpha} & \left.e^{-x} f(x)\right] \\
= & A_{v, \alpha} \frac{e^{-i\left(\frac{\pi}{4}-\frac{\alpha}{4}\right)}}{\sqrt{2 \pi \sin \frac{\alpha}{2}}} \int_{0}^{\infty} F_{\frac{\alpha}{2}}(y) \int_{0}^{\infty} e^{-\left(1+i y \csc \frac{\alpha}{2}\right) x} x J_{v}\left(\frac{x y}{\sin \frac{\alpha}{2}}\right) d x d y \\
= & A_{v, \alpha} \frac{e^{-i\left(\frac{\pi}{4}-\frac{\alpha}{4}\right)}}{\sqrt{2 \pi \sin \frac{\alpha}{2}}} \int_{0}^{\infty} F_{\frac{\alpha}{2}}(y)\left(1+2 i y \csc \frac{\alpha}{2}\right)^{-\frac{3}{2}} \\
& \cdot\left(\left(1+i y \csc \frac{\alpha}{2}\right)+v\left(1+2 i y \csc \frac{\alpha}{2}\right)^{\frac{1}{2}}\right) \\
& \cdot\left(\frac{y}{\left|\sin \frac{\alpha}{2}\right|\left(1+i y \csc \frac{\alpha}{2}\right)\left(1+2 i y \csc \frac{\alpha}{2}\right)^{\frac{1}{2}}}\right)^{v} d y \\
= & \frac{e^{i\left[v\left(\frac{\pi}{2}-\frac{\alpha}{2}\right)+\frac{\pi}{4}-\frac{\alpha}{4}\right]}}{\left|\sin \frac{\alpha}{2}\right|^{v+\frac{3}{2}} \sqrt{2 \pi}} \int_{0}^{\infty} g(y) F_{\frac{\alpha}{2}}(y) d y .
\end{aligned}
$$

Therefore,

$$
\begin{aligned}
\left|H_{v}^{\alpha}\left[e^{-x} f(x)\right]\right|^{2} & \leq\left|C_{\alpha}\right|^{2} \int_{0}^{\infty}|g(y)|^{2}\left|F_{\frac{\alpha}{2}}(y)\right|^{2} d y \\
& \leq\left|C_{\alpha}\right|^{2}\left\|g^{2}\right\|_{\infty} \int_{0}^{\infty}\left|F_{\frac{\alpha}{2}}(y)\right|^{2} d y \\
& \leq\left|C_{\alpha}\right|^{2}\left\|g^{2}\right\|_{\infty}\|f\|_{2}
\end{aligned}
$$

by using the Parseval relation (1.5). 
Theorem 1 Let $f$ be an almost periodic function and let $\alpha$ be an angle where $\cot \frac{\alpha}{2}>0$ and $\alpha \neq k \pi, k$ is an integer. Then the FrHT off is a strong limit power function in $y$ if $0<x<\frac{2 \lambda_{k}}{\cot \frac{\alpha}{2}}$.

Proof Let $f(x)=\sum_{k=1}^{n} a_{k} e^{i \lambda_{k} x}$ be a trigonometric polynomial where $0<x<\frac{2 \lambda_{k}}{\cot \frac{\alpha}{2}}$. Then

$$
\begin{aligned}
\mathcal{H}_{\nu}^{\alpha}[f](y)= & \frac{e^{i(v+1)\left(\frac{\pi}{2}-\frac{\alpha}{2}\right)}}{\sin \frac{\alpha}{2}} \int_{0}^{\infty} x e^{-\frac{i}{2}\left(x^{2}+y^{2}\right) \cot \frac{\alpha}{2}} \sum_{k=1}^{n} a_{k} e^{i \lambda_{k} x} J_{\nu}\left(\frac{x y}{\sin \frac{\alpha}{2}}\right) d x \\
= & A_{v, \alpha} \sum_{k=1}^{n} a_{k} \int_{0}^{\infty} x e^{-\frac{i}{2}\left(x^{2}+y^{2}\right) \cot \frac{\alpha}{2}+i \lambda_{k} x} \sum_{n=0}^{\infty} \frac{(-1)^{n}\left(\frac{x y}{2 \sin \frac{\alpha}{2}}\right)^{v+2 n}}{n !(v+n) !} \\
= & A_{v, \alpha} \sum_{k=1}^{n} a_{k} \sum_{n=0}^{\infty} \frac{(-1)^{n} y^{\nu+2 n}}{n !(v+n) !\left(2 \sin \frac{\alpha}{2}\right)^{v+2 n}} e^{-\frac{i}{2} y^{2} \cot \frac{\alpha}{2}} \\
& \cdot \int_{0}^{\infty} x^{\nu+2 n+1} e^{-\frac{i}{2} x^{2} \cot \frac{\alpha}{2}+i \lambda_{k} x} d x \\
= & A_{v, \alpha} \sum_{k=1}^{n} a_{k} \sum_{n=0}^{\infty} \frac{(-1)^{n} y^{v+2 n}}{n !(v+n) !\left(2 \sin \frac{\alpha}{2}\right)^{v+2 n}} e^{-\frac{i}{2} y^{2} \cot \frac{\alpha}{2}+\frac{i}{2} \frac{\lambda_{k}^{2}}{\cot \frac{\alpha}{2}}} \\
& \cdot \int_{0}^{\infty} x^{\nu+2 n+1} e^{-\frac{i}{2} \cot \frac{\alpha}{2}\left(x-\frac{\lambda_{k}}{\cot \frac{\alpha}{2}}\right)^{2}} d x .
\end{aligned}
$$

Using the substitution $u=x-\frac{\lambda_{k}}{\cot \frac{\alpha}{2}}$, we write

$$
\begin{aligned}
\int_{0}^{\infty} & x^{v+2 n+1} e^{-\frac{i}{2} \cot \frac{\alpha}{2}\left(x-\frac{\lambda_{k}}{\cot \frac{\alpha}{2}}\right)^{2}} d x \\
\quad & \int_{0}^{\infty}\left(u+\frac{\lambda_{k}}{\cot \frac{\alpha}{2}}\right)^{v+2 n+1} e^{-\frac{i}{2} \cot \frac{\alpha}{2} u^{2}} d u \\
& =\int_{0}^{\infty} \sum_{l=0}^{\infty}\left(\begin{array}{c}
v+2 n+1 \\
l
\end{array}\right) u^{l}\left(\frac{\lambda_{k}}{\cot \frac{\alpha}{2}}\right)^{v+2 n+1-l} e^{-\frac{i}{2} \cot \frac{\alpha}{2} u^{2}} d u
\end{aligned}
$$

since the binomial series converges for $0<x<\frac{2 \lambda_{k}}{\cot \frac{\alpha}{2}}$. This enables us to use Fubini's theorem and we have

$$
\begin{aligned}
& \int_{0}^{\infty} x^{\nu+2 n+1} e^{-\frac{i}{2} \cot \frac{\alpha}{2}\left(x-\frac{\lambda_{k}}{\cot \frac{\alpha}{2}}\right)^{2}} d x \\
& =\sum_{l=0}^{\infty}\left(\begin{array}{c}
\nu+2 n+1 \\
l
\end{array}\right)\left(\frac{\lambda_{k}}{\cot \frac{\alpha}{2}}\right)^{\nu+2 n+1-l} \int_{0}^{\infty} u^{l} e^{-\frac{i}{2} \cot \frac{\alpha}{2} u^{2}} d u .
\end{aligned}
$$

By [26], we know that

$$
\int_{0}^{\infty} u^{l} e^{-\frac{i}{2} \cot \frac{\alpha}{2} u^{2}} d u= \begin{cases}\frac{1}{2} \Gamma\left(\frac{l+1}{2}\right) /\left(\frac{i}{2} \cot \frac{\alpha}{2}\right)^{\frac{l+1}{2}}, & l>-1, \\ \frac{(2 m-1) ! !}{2^{m+1}\left(\frac{i}{2} \cot \frac{\alpha}{2}\right)^{-m} \sqrt{\frac{2 \pi}{i \cot \frac{\alpha}{2}}},} & l=2 m, m \text { integer } \\ m ! / 2\left(\frac{i}{2} \cot \frac{\alpha}{2}\right)^{m+1}, & l=2 m+1, m \text { integer }\end{cases}
$$


since $\cot \frac{\alpha}{2}>0$, we find a constant $C_{m, n}\left(\alpha, \lambda_{k}\right)$ as the solution of (2.4). So

$$
\begin{aligned}
\mathcal{H}_{\nu}^{\alpha}[f](y)= & \frac{e^{i(v+1)\left(\frac{\pi}{2}-\frac{\alpha}{2}\right)}}{2^{v}\left(\sin \frac{\alpha}{2}\right)^{v+1}} \sum_{n=0}^{\infty} \frac{(-1)^{n}}{n !(v+n) !\left(2 \sin \frac{\alpha}{2}\right)^{2 n}} y^{\nu+2 n} \\
& \cdot \sum_{k=1}^{n} a_{k} C_{m, n}\left(\alpha, \lambda_{k}\right) e^{-\frac{i}{2} y^{2} \cot \frac{\alpha}{2}+\frac{i}{2} \frac{\lambda_{k}^{2}}{\cot \frac{\alpha}{2}}}
\end{aligned}
$$

which shows that it is a generalized trigonometric polynomial in $y$.

If $f$ is a general almost periodic function, then there exists a sequence $\left(f_{n}\right)$ of trigonometric polynomials where $f_{n} \rightarrow f$ uniformly. Since $\mathcal{H}_{v, n}^{\alpha}(y) \in \operatorname{Gtrig}(\mathbb{R})$, we get $\mathcal{H}_{v}^{\alpha}(y) \in$ $\mathcal{S L P}(\mathbb{R})$.

Thus it is sufficient to verify that, if $\left\|f_{n}-f\right\|_{\infty} \rightarrow 0$, then $\left\|\mathcal{H}_{v, n}^{\alpha}(y)-\mathcal{H}_{v}^{\alpha}(y)\right\|_{L_{\infty}(y)} \rightarrow 0$.

Using the definition of the FrHT, it is easy to see that

$$
\left|\mathcal{H}_{\nu}^{\alpha}(y)\right| \leq \int_{0}^{\infty}\left|f(x)\|x\| J_{v}\right| d x
$$

and

$$
\left|\mathcal{H}_{v, n}^{\alpha}(y)-\mathcal{H}_{v}^{\alpha}(y)\right| \leq \int_{0}^{\infty}\left|f_{n}(x)-f(x)\|x\| J_{\nu}\right| d x \rightarrow 0
$$

when $0<x<\frac{2 \lambda_{k}}{\cot \frac{\alpha}{2}}$. Since

$$
\left\|\mathcal{H}_{v, n}^{\alpha}(y)-\mathcal{H}_{v}^{\alpha}(y)\right\|_{L_{\infty}(y)}=\sup \left|\mathcal{H}_{v, n}^{\alpha}(y)-\mathcal{H}_{v}^{\alpha}(y)\right| \rightarrow 0
$$

the result follows.

Theorem 2 Let $f$ be an almost periodic function and $S_{n ; a, b}^{v}(x)=2^{a / 2} S_{n}^{v}\left(2^{a} x-b\right)$ be an orthogonal basis. Then there exist constants $A, B>0$, such that

$$
A\|f\|_{\mathcal{A P}}^{2} \leq \sum_{a \in \mathbb{Z}} \lim _{N \rightarrow \infty} \frac{1}{2 N+1} \sum_{b=-N}^{N} \mid\left\langle f,\left.S_{n ; a, b}^{v}\right|^{2} \leq B\|f\|_{\mathcal{A P}}^{2}\right.
$$

Proof We have

$$
\begin{aligned}
\left\langle f, S_{n ; a, b}^{v}\right\rangle & =2^{a / 2} C \sum_{k=0}^{n} \bar{c}_{k} \int_{-\infty}^{\infty} f(x) \overline{\psi_{k+\frac{v}{2}}\left(2^{a+1} x-b\right)} d x \\
& =\frac{C}{\sqrt{2}} \sum_{k=0}^{n} \bar{c}_{k}\left\langle f, \psi_{k+\frac{v}{2} ; a+1, b}\right\rangle \\
& =\frac{C}{\sqrt{2}} \sum_{k=0}^{n} \bar{c}_{k}\left(W_{\psi_{k+\frac{1}{2}}} f\right)(b, a+1),
\end{aligned}
$$

where $\left(W_{\psi} f\right)(b, a)=\left\langle f, \psi_{a, b}\right\rangle$ shows the wavelet transform of $f$. Since $f$ is an almost periodic function, $W_{\psi_{k+\frac{1}{2}}} f$ is an almost periodic function in $b$ as well [27]. Therefore 
$\left\{\left\langle f, S_{n ; a, b}^{v}\right\rangle\right\}_{b=-\infty}^{\infty}$ is a sequence of almost periodic functions. If $f$ is a trigonometric polynomial $f(x)=\sum_{\ell=1}^{K} a_{\ell} e^{i \lambda_{\ell} x}$, we get

$$
\begin{aligned}
\left\langle f, S_{n ; a, b}^{\nu}\right\rangle & =2^{a / 2} C \sum_{k=0}^{n} \bar{c}_{k} \sum_{\ell=1}^{K} a_{l} \int_{-\infty}^{\infty} \overline{\psi_{k+\frac{v}{2}}\left(2^{a+1} x-b\right)} e^{i \lambda_{\ell} x} d x \\
& =2^{-\left(\frac{a}{2}+1\right)} C \sum_{k=0}^{n} \bar{c}_{k} \sum_{\ell=1}^{K} a_{l} e^{\frac{i \lambda_{\ell} b}{2^{a+1}}} \overline{\hat{\psi}_{k+\frac{v}{2}}\left(\frac{\lambda_{\ell}}{2^{a+1}}\right)} .
\end{aligned}
$$

Therefore,

$$
\begin{aligned}
\lim _{N \rightarrow \infty} & \frac{1}{2 N+1} \sum_{b=-N}^{N} \sum_{a=-\infty}^{\infty} \mid\left\langle f,\left.S_{n ; a, b}^{\nu}\right|^{2}\right. \\
= & C^{2}\left[\sum_{k=0}^{n}\left|c_{k}\right|^{2}\left[\sum_{\ell=1}^{K}\left|a_{\ell}\right|^{2} h_{k}\left(\lambda_{\ell}\right)+\sum^{\prime}{ }^{\prime} a_{m} \bar{a}_{\ell} j_{k}\left(\lambda_{\ell}, \lambda_{m}\right)\right]\right. \\
& \left.+\sum_{i=0}^{n} \sum_{k=0, k \neq i}^{n} \bar{c}_{i} c_{k}\left[\sum_{\ell=1}^{K}\left|a_{\ell}\right|^{2} h_{i, k}\left(\lambda_{\ell}\right)+\sum^{\prime} a_{m} \bar{a}_{\ell} j_{i, k}\left(\lambda_{\ell}, \lambda_{m}\right)\right]\right],
\end{aligned}
$$

where the sum $\sum^{\prime}$ is taken over those $\ell, m$ such that $\lambda_{m}-\lambda_{\ell}$ is a (nonzero) multiple of $\frac{\pi 2^{a+2}}{b}$ with

$$
\begin{aligned}
& h_{k}\left(\lambda_{\ell}\right)=\sum_{a=-\infty}^{\infty} 2^{-(a+2)}\left|\hat{\psi}_{k+\frac{v}{2}}\left(\frac{\lambda_{l}}{2^{a+1}}\right)\right|^{2} \\
& j_{k}\left(\lambda_{\ell}, \lambda_{m}\right)=\sum_{a=-\infty}^{\infty} 2^{-(a+2)} \hat{\psi}_{k+\frac{v}{2}}\left(\frac{\lambda_{\ell}}{2^{a+1}}\right) \overline{\hat{\psi}_{k+\frac{v}{2}}\left(\frac{\lambda_{m}}{2^{a+1}}\right)} \\
& h_{i, k}\left(\lambda_{\ell}\right)=\sum_{a=-\infty}^{\infty} 2^{-(a+2)} \overline{\hat{\psi}_{i+\frac{v}{2}}\left(\frac{\lambda_{\ell}}{2^{a+1}}\right)} \hat{\psi}_{k+\frac{v}{2}}\left(\frac{\lambda_{\ell}}{2^{a+1}}\right)
\end{aligned}
$$

and

$$
j_{i, k}\left(\lambda_{\ell}, \lambda_{m}\right)=\sum_{a=-\infty}^{\infty} 2^{-(a+2)} \overline{\hat{\psi}_{i+\frac{v}{2}}\left(\frac{\lambda_{m}}{2^{a+1}}\right)} \hat{\psi}_{k+\frac{v}{2}}\left(\frac{\lambda_{\ell}}{2^{a+1}}\right) .
$$

In this case,

$$
\begin{aligned}
& \left|\sum^{\prime} a_{m} \bar{a}_{\ell} j_{k}\left(\lambda_{\ell}, \lambda_{m}\right)\right| \\
& =\mid \sum_{\lambda \in \mathbb{R}} \sum_{s \in \mathbb{Z} \backslash\{0\}} a_{\lambda} \bar{a}_{\lambda+\frac{\pi 2^{a+2} 2_{s}}{b}} \sum_{a=-\infty}^{\infty} 2^{-(a+2)} \hat{\psi}_{k+\frac{v}{2}}\left(\frac{\lambda}{2^{a+1}}\right) \overline{\hat{\psi}_{k+\frac{v}{2}}\left(\frac{\lambda}{2^{a+1}}+\frac{2 \pi s}{b}\right) \mid} \\
& \leq \sum_{s \in \mathbb{Z} \backslash\{0\}}\left(\sum_{a=-\infty}^{\infty} \sum_{\lambda \in \mathbb{R}}\left|a_{\lambda}\right|^{2}\left|\hat{\psi}_{k+\frac{v}{2}}\left(\frac{\lambda}{2^{a+1}}\right)\right|\left|\hat{\psi}_{k+\frac{v}{2}}\left(\frac{\lambda}{2^{a+1}}+\frac{2 \pi s}{b}\right)\right|\right)^{1 / 2} \\
& \quad \times\left(\sum_{a=-\infty}^{\infty} \sum_{\lambda \in \mathbb{R}}\left|a_{\lambda+\frac{\pi 2^{a+2} s_{s}}{b}}\right|^{2}\left|\hat{\psi}_{k+\frac{v}{2}}\left(\frac{\lambda}{2^{a+1}}\right)\right|\left|\hat{\psi}_{k+\frac{v}{2}}\left(\frac{\lambda}{2^{a+1}}+\frac{2 \pi s}{b}\right)\right|\right)^{1 / 2}
\end{aligned}
$$




$$
\begin{aligned}
\leq & \sum_{s \in \mathbb{Z} \backslash\{0\}}\left(\sum_{a=-\infty}^{\infty} \sum_{\lambda \in \mathbb{R}}\left|a_{\lambda}\right|^{2}\left|\hat{\psi}_{k+\frac{v}{2}}\left(\frac{\lambda}{2^{a+1}}\right)\right|\left|\hat{\psi}_{k+\frac{v}{2}}\left(\frac{\lambda}{2^{a+1}}+\frac{2 \pi s}{b}\right)\right|\right)^{1 / 2} \\
& \times\left(\sum_{a=-\infty}^{\infty} \sum_{\lambda \in \mathbb{R}}\left|a_{\lambda}\right|^{2}\left|\hat{\psi}_{k+\frac{v}{2}}\left(\frac{\lambda}{2^{a+1}}-\frac{2 \pi s}{b}\right)\right|\left|\hat{\psi}_{k+\frac{v}{2}}\left(\frac{\lambda}{2^{a+1}}\right)\right|\right)^{1 / 2} \\
\leq & \sum_{\lambda \in \mathbb{R}}\left|a_{\lambda}\right|^{2} \sum_{s \in \mathbb{Z} \backslash\{0\}}\left(\Gamma_{1}(s) \Gamma_{1}(-s)\right)^{1 / 2},
\end{aligned}
$$

where $\Gamma_{1}(s)=\sup _{\lambda \in \mathbb{R}} \sum_{a \in \mathbb{Z}}\left|\hat{\psi}_{k+\frac{v}{2}}\left(\frac{\lambda}{2^{a+1}}\right)\right|\left|\hat{\psi}_{k+\frac{v}{2}}\left(\frac{\lambda}{2^{a+1}}+\frac{2 \pi s}{b}\right)\right|$ and let us take

$$
\begin{aligned}
& A_{1}=\inf _{\lambda \in \mathbb{R}} \sum_{a \in \mathbb{Z}}\left|\hat{\psi}_{k+\frac{v}{2}}\left(\frac{\lambda}{2^{a+1}}\right)\right|^{2}-\sum_{s \in \mathbb{Z} \backslash\{0\}}\left(\Gamma_{1}(s) \Gamma_{1}(-s)\right)^{1 / 2}>0, \\
& B_{1}=\sup _{\lambda \in \mathbb{R}} \sum_{a \in \mathbb{Z}}\left|\hat{\psi}_{k+\frac{v}{2}}\left(\frac{\lambda}{2^{a+1}}\right)\right|^{2}+\sum_{s \in \mathbb{Z} \backslash\{0\}}\left(\Gamma_{1}(s) \Gamma_{1}(-s)\right)^{1 / 2}<\infty .
\end{aligned}
$$

Similarly, we get the inequality

$$
\left|\sum^{\prime} a_{m} \bar{a}_{\ell} j_{i, k}\left(\lambda_{\ell}, \lambda_{m}\right)\right| \leq \sum_{\lambda \in \mathbb{R}}\left|a_{\lambda}\right|^{2} \sum_{s \in \mathbb{Z} \backslash\{0\}}\left(\Gamma_{2}(s) \Gamma_{2}(-s)\right)^{1 / 2},
$$

where $\Gamma_{2}(s)=\sup _{\lambda \in \mathbb{R}} \sum_{a \in \mathbb{Z}}\left|\hat{\psi}_{i+\frac{v}{2}}\left(\frac{\lambda}{2^{a+1}}\right)\right|\left|\hat{\psi}_{k+\frac{v}{2}}\left(\frac{\lambda}{2^{a+1}}+\frac{2 \pi s}{b}\right)\right|$ and we take

$$
\begin{aligned}
& A_{2}=\inf _{\lambda \in \mathbb{R}} \sum_{a \in \mathbb{Z}}\left|\hat{\psi}_{i+\frac{v}{2}}\left(\frac{\lambda}{2^{a+1}}\right)\right|\left|\hat{\psi}_{k+\frac{v}{2}}\left(\frac{\lambda}{2^{a+1}}\right)\right|-\sum_{s \in \mathbb{Z} \backslash\{0\}}\left(\Gamma_{2}(s) \Gamma_{2}(-s)\right)^{1 / 2}>0, \\
& B_{2}=\sup _{\lambda \in \mathbb{R}} \sum_{a \in \mathbb{Z}}\left|\hat{\psi}_{i+\frac{v}{2}}\left(\frac{\lambda}{2^{a+1}}\right)\right|\left|\hat{\psi}_{k+\frac{v}{2}}\left(\frac{\lambda}{2^{a+1}}\right)\right|+\sum_{s \in \mathbb{Z} \backslash\{0\}}\left(\Gamma_{2}(s) \Gamma_{2}(-s)\right)^{1 / 2}<\infty .
\end{aligned}
$$

Using (2.7) and the constants $A_{1}, B_{1}, A_{2}, B_{2}$, we get the inequality (2.6) for the trigonometric polynomials. Then we find the result for almost periodic functions by a standard approximation.

Theorem 3 Let $f$ be an almost periodic function and let $\alpha$ be an angle where $\cot \frac{\alpha}{2}>0$.

Then the BWT off is a strong limit power function in $y$.

Proof Let $f(t)=\sum_{k=1}^{n} a_{k} e^{i \lambda_{k} t}$ be a trigonometric polynomial. Then

$$
\begin{aligned}
\left(B_{\psi} f\right)(b, a) & =\int_{0}^{\infty} f(t) \overline{\psi_{b, a}(t)} d \sigma(t)=\int_{0}^{\infty} \sum_{k=1}^{n} a_{k} e^{i \lambda_{k} t} \overline{\psi_{b, a}(t)} \frac{(2 \gamma+1) t^{2 \gamma}}{2^{\gamma+1 / 2} \Gamma\left(\gamma+\frac{3}{2}\right)} d t \\
& =\frac{(2 \gamma+1)}{2^{\gamma+1 / 2} \Gamma\left(\gamma+\frac{3}{2}\right)} \sum_{k=1}^{n} a_{k} \int_{0}^{\infty} t^{2 \gamma} e^{i \lambda_{k} t} \overline{\psi_{b, a}(t)} d t \\
& =\frac{(2 \gamma+1)}{2^{\gamma+1 / 2} \Gamma\left(\gamma+\frac{3}{2}\right)} \sum_{k=1}^{n} a_{k} e^{-i \lambda_{k} v} \int_{v}^{\infty}(u-v)^{2 \gamma} e^{i \lambda_{k} u} \overline{\psi_{b, a}(u-v)} d u \\
& =\frac{(2 \gamma+1)}{2^{\gamma+1 / 2} \Gamma\left(\gamma+\frac{3}{2}\right)} \sum_{k=1}^{n} a_{k} e^{-i \lambda_{k} v} \overline{W_{\phi_{b, a}}\left(v, \lambda_{k}\right)},
\end{aligned}
$$


where $W_{\phi_{b, a}}$ is the wave packet transform [28] with respect to the Bessel wavelet. Hence $\left(B_{\psi} f\right)(b, a)$ is a trigonometric polynomial in $v$.

For a general almost periodic function $f$ we take a sequence of trigonometric polynomials $\left(f_{n}\right)$ such that $\left\|f_{n}-f\right\|_{\infty} \rightarrow 0$, and it will be sufficient to verify that $\left\|B_{\psi} f_{n}-B_{\psi} f\right\|_{L^{\infty}(v)} \rightarrow$ 0 . Using the solutions in [20], we have

$$
\begin{aligned}
\left\|B_{\psi} f_{n}-B_{\psi} f\right\|_{L^{\infty}(v)} & =\left\|\left(f_{n}-f\right) \sharp \phi\right\|_{L^{\infty}(v)} \\
& =\underset{v}{\operatorname{ess} \sup }\left|\int_{0}^{\infty}\left(f_{n}(u-v)-f(u-v)\right) \overline{\psi_{b, a}(u-v)} d \sigma(u)\right| \\
& \leq\left\|f_{n}-f\right\|_{\infty}\left\|\psi_{b, a}\right\|_{1},
\end{aligned}
$$

which gives the desired result.

\section{Competing interests}

The author declares that she has no competing interests.

Received: 8 July 2015 Accepted: 26 November 2015 Published online: 09 December 2015

\section{References}

1. Arfken, GB, Weber, HJ, Harris, FE: Mathematical Methods for Physicists: A Comprehensive Guide. Academic Press, New York (2012)

2. Sneddon, IN: The Use of Integral Transforms. McGraw-Hill, New York (1972)

3. Tuan, VK, Saigo, M: Convolution of Hankel transform and its application to an integral involving Bessel functions of first kind. Int. J. Math. Math. Sci. 18(3), 545-550 (1995)

4. Kerr, FH: A fractional power theory for Hankel transforms. J. Math. Anal. Appl. 158, 114-123 (1991)

5. Namias, V: Fractionalization of Hankel transform. J. Inst. Math. Appl. 26, 187-197 (1980)

6. Prasad, A, Mahato, KL: The fractional Hankel wavelet transformation. Asian-Eur. J. Math. 8(2), 1550030 (2015)

7. Sheppard, CJR, Larkin, KG: Similarity theorems for fractional Fourier transforms and fractional Hankel transforms. Opt. Commun. 154, 173-178 (1998)

8. Taywade, RD, Gudadhe, AS, Mahalle, VN: Generalized operational relations and properties of fractional Hankel transform. Sci. Rev. Chem. Commun. 2(3), 282-288 (2012)

9. Taywade, RD, Gudadhe, AS, Mahalle, VN: Initial and final value theorem on fractional Hankel transform. IOSR J. Math. 5, 36-39 (2013)

10. Namias, $\mathrm{V}$ : The fractional order Fourier transform and its application to quantum mechanics. J. Inst. Math. Appl. 25, 241-265 (1980)

11. Ozaktas, HM, Zalvesky, Z, Kuntay, MA: The Fractional Fourier Transform with Applications in Optics and Signal Processing. Wiley, Chichester (2001)

12. Pei, S-C: Fractional cosine, sine, and Hartley transforms. IEEE Trans. Signal Process. 50(7), 1661-1680 (2012)

13. Pathak, RS, Dixit, MM: Continuous and discrete Bessel wavelet transforms. J. Comput. Appl. Math. 160(1-2), 241-250 (2003)

14. Prasad, A, Mahato, A, Singh, VK, Dixit, MM: The continuous fractional Bessel wavelet transformation. Bound. Value Probl. 2013, 40 (2013)

15. Upadhyay, SK, Yadav, RN, Debnath, L: On continuous Bessel wavelet transformation associated with the Hankel-Hausdorff operator. Integral Transforms Spec. Funct. 23(5), 315-323 (2012)

16. Poularikas, AD: Transforms and Applications Handbook. CRC Press, Boca Raton (2010)

17. Gudadhe, AS, Taywade, RD, Mahalle, VN: Namias fractional Hankel transform in the Zemanian space. Glob. J. Math. Sci.: Theory Pract. 5(3), 145-151 (2013)

18. Shen, L-C: Orthogonal polynomials on the unit circle associated with the Laguerre polynomials. Proc. Am. Math. Soc. 129(3), 873-879 (2001)

19. Gradshteyn, IS, Ryzhik, IM: Tables of Integrals, Series and Products. Academic Press, New York (2000)

20. Pathak, RS, Upadhyay, SK, Pandey, RS: The Bessel wavelet convolution product. Rend. Semin. Mat. (Torino) 69(3), 267-279 (2011)

21. Besicovitch, AS: Almost Periodic Functions. Dover, Cambridge (1954)

22. Corduneanu, C: Almost Periodic Functions. Chelsea Publishing Company, New York (1989)

23. Zhang, C: Strong limit power functions. Studia Sci. Math. Hung. 12(3), 291-307 (2006)

24. Zhang, C, Meng, C: Two new spaces of vector-valued limit power functions. Studia Sci. Math. Hung. 44(4), 423-443 (2007)

25. Gudadhe, AS, Taywade, RD, Mahalle, VN: A relation between fractional Hankel transform and other integral transforms of fractional order. Asian J. Curr. Eng. Maths 2(5), 301-305 (2013)

26. Jeffrey, A, Zwillinger, D: Table of Integrals, Series, and Products. Academic Press, New York (2007)

27. Ünalmış Uzun, B: Generalized frames in the space of strong limit power functions. Mediterr. J. Math. 10(1), 321-332 (2013)

28. Kato, K, Kobayashi, M, Ito, S: Remark on wave front sets of solutions to Schrödinger equation of a free particle and a harmonic oscillator. SUT J. Math. 47(2), 175-183 (2011) 